\title{
Optimal energy for cell radiosensitivity enhancement by gold nanoparticles using synchrotron-based monoenergetic photon beams
}

This article was published in the following Dove Press journal:

International Journal of Nanomedicine

19 May 2014

Number of times this article has been viewed

\author{
Wan Nordiana Rahman ${ }^{1,2}$ \\ Stéphanie Corde ${ }^{3,4}$ \\ Naoto Yagi ${ }^{5}$ \\ Siti Aishah Abdul Aziz' \\ Nathan Annabell ${ }^{2}$ \\ Moshi Geso ${ }^{2}$ \\ 'School of Health Sciences, Universiti \\ Sains Malaysia, Kelantan, Malaysia; \\ ${ }^{2}$ Division of Medical Radiation, School \\ of Medical Sciences, Royal Melbourne \\ Institute of Technology, Bundoora, VIC, \\ ${ }^{3}$ Radiation Oncology, Prince of Wales \\ Hospital, High Street, Randwick, \\ ${ }^{4}$ Centre for Medical Radiation Physics, \\ University of Wollongong, Wollongong, \\ NSW, Australia; ${ }^{5}$ Japanese Synchrotron \\ Radiation Research Institute, Sayo-gun, \\ Hyogo, Japan
}

\begin{abstract}
Gold nanoparticles have been shown to enhance radiation doses delivered to biological targets due to the high absorption coefficient of gold atoms, stemming from their high atomic number $(Z)$ and physical density. These properties significantly increase the likelihood of photoelectric effects and Compton scattering interactions. Gold nanoparticles are a novel radiosensitizing agent that can potentially be used to increase the effectiveness of current radiation therapy techniques and improve the diagnosis and treatment of cancer. However, the optimum radiosensitization effect of gold nanoparticles is strongly dependent on photon energy, which theoretically is predicted to occur in the kilovoltage range of energy. In this research, synchrotron-generated monoenergetic X-rays in the 30-100 keV range were used to investigate the energy dependence of radiosensitization by gold nanoparticles and also to determine the photon energy that produces optimum effects. This investigation was conducted using cells in culture to measure dose enhancement. Bovine aortic endothelial cells with and without gold nanoparticles were irradiated with X-rays at energies of 30, 40, 50, 60, 70, 81, and $100 \mathrm{keV}$. Trypan blue exclusion assays were performed after irradiation to determine cell viability. Cell radiosensitivity enhancement was indicated by the dose enhancement factor which was found to be maximum at $40 \mathrm{keV}$ with a value of 3.47. The dose enhancement factor obtained at other energy levels followed the same direction as the theoretical calculations based on the ratio of the mass energy absorption coefficients of gold and water. This experimental evidence shows that the radiosensitization effect of gold nanoparticles varies with photon energy as predicted from theoretical calculations. However, prediction based on theoretical assumptions is sometimes difficult due to the complexity of biological systems, so further study at the cellular level is required to fully characterize the effects of gold nanoparticles with ionizing radiation.
\end{abstract}

Keywords: gold nanoparticles, radiotherapy, monoenergetic synchrotron radiation, radiosensitizers, in vitro

\section{Introduction}

Gold nanoparticles are of increasing interest in a variety of biomedical applications due to their unique properties. These applications include use as a radiosensitizer to enhance delivery of a lethal radiation dose to tumor tissue while minimizing harmful radiation to normal tissue. Gold nanoparticles composed of atoms with a high atomic number $(Z)$ have been the target of extensive investigation for their potential application in the treatment of cancer using ionizing radiation. ${ }^{1-5}$ Gold nanoparticles are well known for their high mass energy absorption coefficient due to their increased probability of photoelectric interaction, which occurs predominantly in the kilovoltage range of energy, ie, below $500 \mathrm{keV}$. This increase in probability of photoelectric absorption results in increased energy being deposited at the target site. ${ }^{1}$ 
The technique of enhancing local tumor control by using high $\mathrm{Z}$ materials to increase the absorbed dose was initially investigated using iodine. Iodine compounds have been used as contrast agents in clinical radiology for a long time. The principle of contrast enhancement by such agents stems from the fact that iodine atoms have a higher atomic number and physical density than most of the atoms present in human body tissue. Therefore, iodine atoms will absorb X-rays more efficiently, leading to more variation in X-ray absorption across the image, leading to enhanced image contrast.

Increases in dose effects as a result of image contrast enhancement in radiographic imaging procedure with iodine contrast agent have been observed in the earlier study. ${ }^{6,7}$ The same properties of the iodine as a high atomic number and high physical density contrast agent were later utilized as a radiation dose enhancement tool in radiotherapy. ${ }^{6,7}$ It was further suggested that, by loading tumors with iodine and using a kilovoltage energy beam, the radiation dose could be concentrated preferentially in tumor tissue due to an increase in local photoelectric interactions. The presence of these iodine-based contrast agents in the tumor during the course of radiotherapy would lead to enhancement of the radiation dose at the target site due to increased absorption of X-rays by the iodine atoms, which in turn would result in generation of free radicals, thereby increasing the likelihood of damage to DNA molecules in tumor cells. In other words, the iodine contrast agent radiosensitizes the target and enhances the dose delivered to the target. However, this was found to be most effective at the lower end of the range of X-ray energy used in radiotherapy. ${ }^{8,9} \mathrm{Current}$ radiotherapy modalities commonly use X-ray energy in the megavoltage range, which is unsuitable for such an application. Investigations of radiosensitization by iodine at high photon energy yields show negligible dose enhancement as compared with kilovoltage photon energy.,

Radiation dose enhancement by inclusion of such heavy atoms in the target is anticipated to be radiation beam energy-dependent. This is supported by the fact that energy deposition by ionizing radiation is energy-dependent and the optimal energy value lies just above the $\mathrm{K}$-edge value of the target atom. However, based on the dose enhancement factor (DEF), this might not be the case when the energy dependence is not linked to the $\mathrm{K}$-edge value. The DEF is defined as the increase in dose due to the presence of heavy atoms in water. It is deduced from the ratio of the mass energy absorption coefficient of the heavy atom compared with that of water. Based on this definition, dependence of DEF on the beam energy is not directly related to the $\mathrm{K}$-edge value of the atoms. ${ }^{10}$ Early studies of iodine atoms indicate that the DEF depends on the beam energy. ${ }^{10}$ However, those studies used radiation sources that were not monoenergetic, such as the $\mathrm{X}$-ray tube. Using such sources of radiation, it is not possible to quantify the beam energy dependence of DEF values.

Studies using iodine with a monoenergetic synchrotron beam have demonstrated that cell radiosensitivity enhancement depends on the photon beam energy as predicted by theoretical calculation, with an experimental maximum at $50 \mathrm{keV}$ and a minimum just below the iodine K-absorption edge. ${ }^{10}$ Studies of polymer gel doped with iodine showed similar variation in dose enhancement when compared with the theoretical calculations and the results of an in vitro study. ${ }^{11}$ This phantom study using normoxic polyacrylamide gel also found that the DEF peaks at $50 \mathrm{keV}^{11}$ These preclinical studies led to the first human clinical trial of iodine together with monochromatic synchrotron energy in the treatment of cancer at the European Synchrotron Radiation Facility. ${ }^{12}$ The first clinical trial using iodine as radiosensitizer fostered the innovation of possible potential of the nanoparticle based radiosensitizer for radiation therapy.

A number of recent studies have assessed the effects of radiosensitization and dose enhancement using gold nanoparticles. ${ }^{13,14}$ The effects of gold nanoparticles on distribution of the radiation dose have been calculated using Monte Carlo simulation with various models. Simulated scenarios are used to generate interaction of individual photons and electrons with gold nanoparticles and predict the amount of energy deposited in the surrounding target. ${ }^{15}$ Many such theoretical calculation studies have demonstrated the potential of kilovoltage irradiation as a better energy choice compared to higher photon energies. ${ }^{13-15}$ The results produced by computational methods vary according to the model used, and need to be clarified by further experimental studies.

Experimental validation of the radiosensitizing effects of gold nanoparticles in vitro and in vivo provides evidence in support of theoretical predictions. These simulation models predict dose enhancement by inclusion of gold atoms as nanoparticles into the target but they do not give details on the physics and biology involved in this irradiation technique. ${ }^{16}$ It should be noted here that these simulation models consider nanoparticles simply as atoms and no consideration is given to the characteristics of the nanoparticles as a unit. In contrast with iodine atoms, nanoparticles may have different mechanisms of radiosensitization due to their small size (eg, a larger surface to volume ratio), which can alter and modify the biological system. Unlike iodine, gold nanoparticles can penetrate into cells easily, and the secondary radiation produced by 
their interaction with ionizing radiation could further damage DNA, leading to cell death. ${ }^{1,2}$ Hainfeld et al pioneered the use of gold nanoparticles as a radiosensitizer in animal models and found significant tumor regression leading to prolonged survival. ${ }^{1,2}$ Studies using cell survival as an outcome measure have been extensively reported following the early animal studies giving variation of results due to different parameters used such as cell lines, radiation energy, and nanoparticle characteristics. $^{3-5,17}$

Butterworth et al recently reviewed the physical and biological mechanisms underlying gold nanoparticle radiosensitization and suggested that the variable results of the studies are attributable to the different gold nanoparticle concentrations and photon energies used. ${ }^{16}$ It is probable that intrinsic cell biological responses are also involved in radiosensitization, over and above the total physical radiation dose delivered. Lechtman et al performed theoretical studies to investigate the implications of photon energy and nanoparticle size, concentration, and localization, and found that dose enhancement by megavoltage energy is impossible unless a gold nanoparticle concentration of around 1,560-1,760 mg/g is present in the target. ${ }^{15}$

Small gold nanoparticles conjugated to tumor-targeted moieties and able to be attached to a DNA structure would be the best option to achieve the optimal dose enhancement effects, together with a large increase in photoelectric absorption in the gold nanoparticles for lower energy sources. ${ }^{15,18}$ However, McMahon et al point out that the difference between $\mathrm{kV}$ and $\mathrm{mV}$ results is less than $2 \%$ and biological factors need to be considered. ${ }^{19}$ Supported by a local effect model, McMahon et al suggest that dose enhancement at higher energy levels can be achieved via production of high LET (linear energy transfer) secondary charged particles from the primary interaction of photons with nanoparticles. ${ }^{19}$ Experimental work by the same group on the energy dependence of gold nanoparticle radiosensitization yielded results that departed significantly from the theoretical calculations, suggesting that the process of radiosensitization is complex and depends on various factors. Recently, a stochastic model of radiation dose enhancement has been described, representing the interactions of incident photons with aggregates of nanoparticles in cells. ${ }^{20}$

In this work, we experimentally determined the optimal energy for dose enhancement by gold nanoparticles using a bovine aortic endothelial cell survival curve based on a viability assay as the measurement end point. A monoenergetic synchrotron beam in the range of 30-100 keV supplied by the BL20XU beam-line at the Spring-8 Biomedical Imaging
Center, Hyogo, Japan, was used to determine the energy dependence of the radiosensitization effects introduced by inclusion of gold nanoparticles.

\section{Materials and methods Gold nanoparticles}

The gold nanoparticles (1.9 nm diameter) used were from Nanoprobes Inc., (AuroVist ${ }^{\circledR}$ gold nanoparticle X-ray contrast agent; Yaphank, NY, USA) and were prepared according to the product specification instructions. They were dissolved in phosphate-buffered saline and the solution was then filtered using the centrifugal tube filter provided. Next, the gold nanoparticle solution was spun in a centrifuge at $10,000 \times \mathrm{g}$ for 5 minutes. The solution was then diluted with complete cell culture medium to the final concentration.

\section{Cell culture}

Bovine aortic endothelial cells were purchased from Cell Applications Inc., (San Diego, CA, USA) and grown in a 75 mL flask (Iwaki and Co., Ltd., Tokyo, Japan) at $37^{\circ} \mathrm{C}$ and in a $5 \% \mathrm{CO}_{2}$ humidified atmosphere until confluence. The cells were cultured in Dulbecco's Modified Eagle's Medium with GlutaMax ${ }^{\mathrm{TM}}-\mathrm{I}, 10 \%$ fetal bovine serum, $100 \mathrm{U} / \mathrm{mL}$ penicillin, and $100 \mu \mathrm{g} / \mathrm{mL}$ streptomycin (all from Gibco, Ghent, Belgium).

\section{Cell irradiation and viability}

Bovine aortic endothelial cells were trypsinized and experiments were conducted with the cell suspension in $1.5 \mathrm{~mL}$ sterile Eppendorf tubes with and without gold nanoparticles. The gold nanoparticles were then added to the cell suspension giving a final concentration of $1 \mathrm{mmol} / \mathrm{L}$ or $0.197 \mathrm{mg} /$ $\mathrm{mL}$. A suspension containing approximately 50,000 cells was irradiated in the vertical position as shown in Figure 1. Cell viability assays were done using the Trypan blue exclusion method immediately after irradiation.

\section{Dosimetry}

The BL20XU beam-line at the Spring-8 Biomedical Imaging Center consists of a monoenergetic X-ray beam with a constant exit double-crystal monochromator system. A configuration of $\mathrm{Si}$ (511) Si (111) was used to test energy levels of $30,40,50,60,70,81$, and $100 \mathrm{keV}$. The prospective dosimetry was performed using a calibrated PTW 30009 pencil-type ionization chamber and electrometer (PTW, Freiburg, Germany). Dose rates calculated in water were kept constant across the energy range (15 cGys per second on average). Beam sizes were measured on 


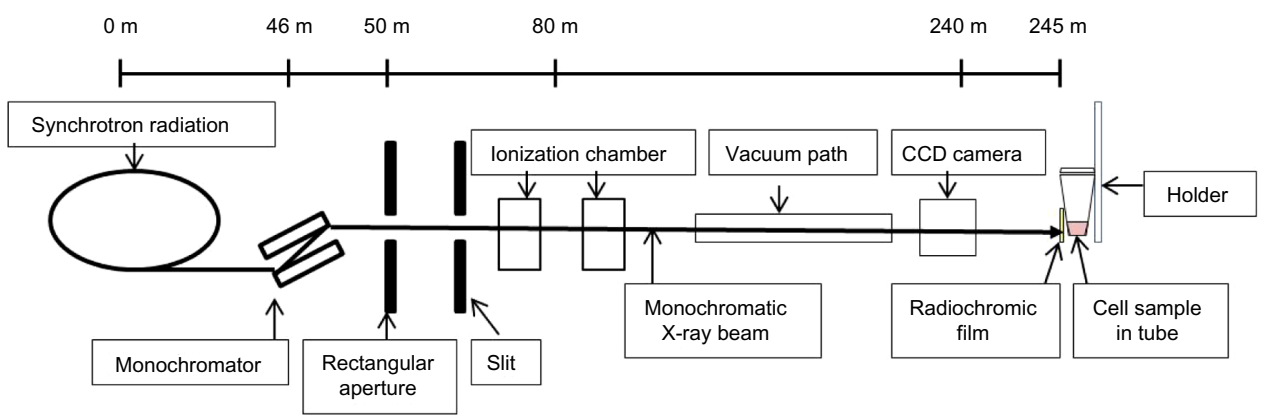

Figure I Schematic diagram of irradiation by the BL20XU beam-line at Spring-8 Biomedical Imaging Center, Hyōgo Prefecture, Japan. Note that the figure is not drawn to scale. Abbreviation: CCD, charge coupled device.

GAFchromic $^{\circledR}$ XRCT2 films (Ashland Specialty Ingredients, Parlin, NJ, USA) and were approximately 0.25 (height) $\times 0.5$ (width) $\mathrm{cm}^{2}$. EBT2 type GAFchromic films were placed on the Eppendorf tubes to verify that no geometric miss occurred during irradiation. To account for potential fluctuations in beam intensity during irradiation of the samples, the doses actually delivered were recalculated a posteriori by measurement with the ionization chamber placed behind the samples.

\section{Statistical analysis}

The data are expressed as the mean \pm standard deviation of three samples. The unpaired Student's $t$-test (Welch corrected) was used to determine the significance of differences between the control and experimental groups. The statistical analysis was performed using GraphPad InStat version 3 for Windows (GraphPad Software, San Diego, CA, USA).

\section{Results}

Theoretical model-based calculations of DEF values for atomic gold as a function of photon energy and mass proportion of gold in the mixture are shown in Figure 2. This DEF calculation is specifically based on an equation for a monoenergetic beam using the ratio of mass energy absorption coefficients obtained from the National Institute of Standard and Technology database. ${ }^{18}$ In this study, DEF values were calculated for different photon energies and various proportions of gold nanoparticles following the same model used by Corde et al for calculation of the dose enhancement produced by iodine. ${ }^{10}$

Our calculations indicate a clear dependence of DEF on photon energy and the relative quantity of gold nanoparticles (Figure 2). The highest DEF value of 160 was obtained with $100 \%$ gold mixtures at a photon energy of $40 \mathrm{keV}$. This optimal energy value is well below the gold K-edge energy, which is $80.7 \mathrm{keV}$. In Figure 2, the DEF increases with increasing energy until it reaches a maximum at $40 \mathrm{keV}$ and gradually decreases until it reaches the gold K-edge energy level of $80.7 \mathrm{keV}$, indicating a sharp increase in dose enhancement. DEF values beyond the K-edge value gradually decrease until almost no enhancement is present at energies above $1 \mathrm{MeV}$. The bell-shaped graph in Figure 2 shows the variation of DEF with photon energy, and clearly indicates that there is an optimal energy where maximum dose enhancement by gold nanoparticles can be achieved. Based on this model, which calculates the mass-energy absorption coefficent ratio, the optimal energy for dose enhancement is not necessarily the K-edge value for the high $\mathrm{Z}$ atomic dose enhancer, because the optimum was found to be clearly inferior to the K-edge energy for gold. This finding is also concordant with the calculations documented by Corde et al for iodine atoms. The model-based predictions demonstrate that maximum dose enhancement occurs at around 40-50 keV, and this has also been demonstrated experimentally. ${ }^{10}$ To investigate the energy dependence of

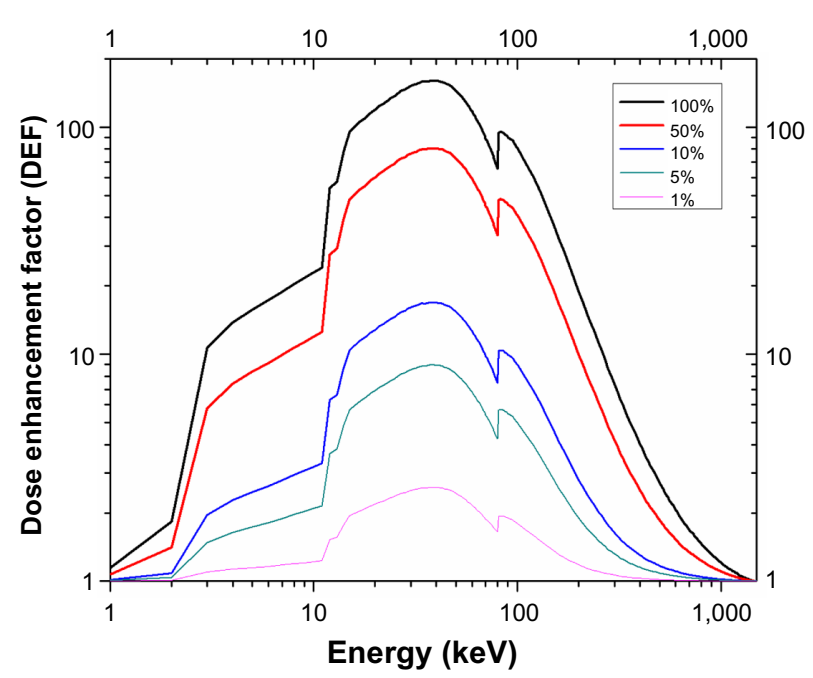

Figure $2 \mathrm{DEF}$ calculated for different percentages of the gold mixture in water at different incident photon energies. The figure shows the dependency of DEF on photon energy and gold concentration (from $1 \%$ to $100 \%$ mass fraction of gold in water). The highest DEF yield is at $40 \mathrm{keV}$.

Abbreviation: DEF, dose enhancement factor. 
gold nanoparticle dose enhancement, monoenergetic photon beams were used to irradiate bovine aortic endothelial cells with and without gold nanoparticles in the culture medium. In this experiment, the effects of subcellular localization of gold nanoparticles were not taken into account.

Figure 3 shows the cell survival curves, all of which indicate a dose modification effect or radiosensitization by gold nanoparticles at all energy levels tested between 30 and
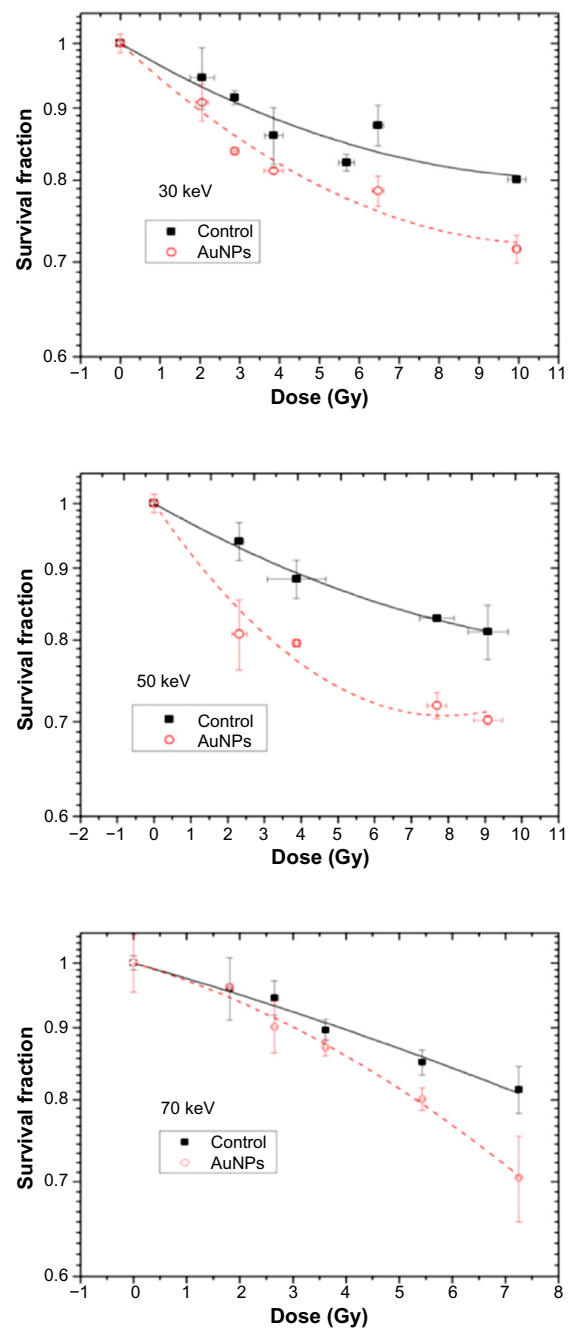

$100 \mathrm{keV}$ and suggest that the amplitude of this phenomenon is energy-dependent. Empirically, the dose enhancement effect of $1 \mathrm{mM}$ gold nanoparticles in bovine aortic endothelial cells is measured at each energy level by estimating the fraction of cells surviving after irradiation with and without gold nanoparticles. The experimental DEF is derived from the ratio of the doses necessary to decrease cell survival to $90 \%$ with and without gold nanoparticles and then comparing these
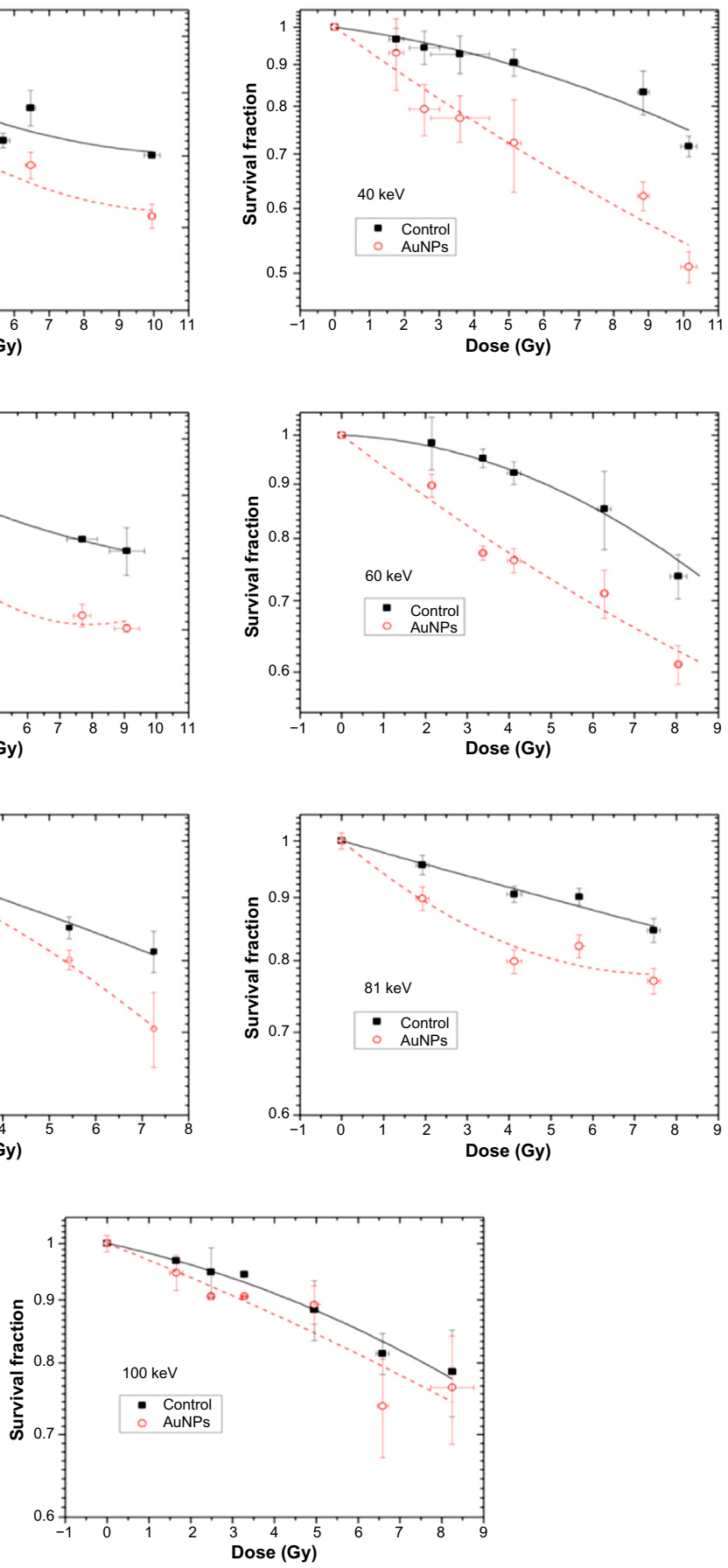

Figure 3 Survival curves for cells irradiated with and without gold nanoparticles were fitted to a linear-quadratic model at different energy levels. Abbreviation: AuNPs, gold nanoparticles. 
with the corresponding calculated values. The cell survival curves were fitted according to the linear-quadratic model and the radiobiological parameters alpha and beta were deduced based on this model. The results obtained for DEF and the alpha and beta values are presented in Table 1 .

As predicted, the DEF deduced from cell survival curves was found to be highest at about $40 \mathrm{keV}$, with a value of 3.47 , followed by $50 \mathrm{keV}$ and $60 \mathrm{keV}$ with values of 2.77 and 3.06 , respectively. These DEF values indicate a more than three-fold enhancement by gold nanoparticles in this range of energies. Less dose enhancement was observed for the extreme energies used in this experiment, despite the fact that a relatively slight increase in dose enhancement exists at gold K-edge energy. At $30 \mathrm{keV}$, a DEF of 1.74 was observed and at 70, 81, and $100 \mathrm{keV}$, the observed DEF values were 1.3, 2.58, and 1.35, respectively. The experimental DEF values obtained at different energies were compared with those based on theoretical calculations, as shown in Figure 4. DEF was calculated at the same mass concentration as that used in the experimental work $(0.197 \mathrm{mg} / \mathrm{mL})$. Comparison between the measured and calculated DEF values shows variation of change is similar in pattern where the DEF peaks at $40 \mathrm{keV}$. However, when the experimental and calculated DEF values are compared for each energy level, the experimental DEF was much higher than the calculated DEF for all radiation energy levels. The differences between the experimental and calculated DEF values are the result of many factors, including experimental geometry, cell radiosensitivity, and amount of radiation dose being absorbed that results in cell death. ${ }^{10}$ The DEF measured at different survival rates can also give different experimental

Table I Radiobiological parameters based on a linear-quadratic model extrapolated from cell survival curves for each energy level with and without gold nanoparticles

\begin{tabular}{|c|c|c|c|c|}
\hline $\begin{array}{l}\text { Energy } \\
(\mathrm{keV})\end{array}$ & $\begin{array}{l}\text { AuNPs } \\
(\mathrm{mmol} / \mathrm{L})\end{array}$ & $\begin{array}{l}\alpha\left(G y^{-1}\right) \\
(\mathrm{SE})\end{array}$ & $\begin{array}{l}\beta\left(G y^{-2}\right) \\
(\mathrm{SE})\end{array}$ & $\begin{array}{l}\text { DEF (at } 90 \% \\
\text { survival) }\end{array}$ \\
\hline \multirow[t]{2}{*}{30} & 0 & $0.038(0.006)$ & $-0.002(0.001)$ & \\
\hline & I & $0.060(0.006)$ & $-0.003(0.001)$ & 1.74 \\
\hline \multirow[t]{2}{*}{40} & 0 & $0.013(0.007)$ & $0.001(0.0003)$ & \\
\hline & I & $0.070(0.012)$ & $-0.001(0.001)$ & 3.47 \\
\hline \multirow[t]{2}{*}{50} & 0 & $0.034(0.003)$ & $-0.001(0.0004)$ & \\
\hline & I & $0.088(0.011)$ & $-0.006(0.001)$ & 2.77 \\
\hline \multirow[t]{2}{*}{60} & 0 & $0.003(0.004)$ & $0.004(0.001)$ & \\
\hline & I & $0.069(0.008)$ & $-0.00 \mathrm{I}(0.00 \mathrm{I})$ & 3.06 \\
\hline \multirow[t]{2}{*}{70} & 0 & $0.025(0.004)$ & $0.001(0.001)$ & \\
\hline & I & $0.026(0.004)$ & $0.003(0.001)$ & 1.3 \\
\hline \multirow[t]{2}{*}{81} & 0 & $0.022(0.005)$ & $-0.000 \mathrm{I}(0.001)$ & \\
\hline & I & $0.065(0.011)$ & $-0.004(0.002)$ & 2.58 \\
\hline \multirow[t]{2}{*}{100} & 0 & $0.016(0.004)$ & $0.002(0.001)$ & \\
\hline & I & $0.031(0.012)$ & $0.001(0.002)$ & 1.35 \\
\hline
\end{tabular}

Abbreviations: AuNPs, gold nanoparticles; DEF, dose enhancement factor; SE, standard error. results. ${ }^{10}$ Therefore, the parameters chosen for analysis are important when evaluating dose enhancement effects.

\section{Discussion}

Analysis of the radiobiological parameters, alpha and beta, computed from a linear-quadratic model, indicates no clear effect of gold nanoparticles on the beta value, but the alpha value follows almost the same trend as the DEF. Figure 5 shows the variation in alpha values for DEF, which have a maximum value at around $40-50 \mathrm{keV}$, where the DEF is also optimum. From this analysis of radiobiological parameters, the low energy range could be the optimal energy level at which to achieve the best radiosensitization by gold nanoparticles, as indicated by the theoretical calculations. This suggests that the alpha value could be used as an indicator, resulting in enhancement of cell death. The alpha value represents the double-strand break given that alpha is the probability of an interaction between two chromosome breaks as a consequence of a single electron set in motion by absorption of X-rays. However, it should be noted that not all energy deposited will contribute to DNA damage through either single-strand breaks or double-strand breaks. The radiation dose will probably be absorbed into other cells and organelles, such as the cytoskeleton, mitochondria, lysosomes, and the plasma membrane, and will also affect cell interaction and signaling. Alterations in the cytoplasmic organelles and cell function caused by ionizing radiation will eventually induce cell death via apoptosis and necrosis. ${ }^{21} \mathrm{~A}$ study by McMahon et al of dose enhancement by gold nanoparticles based on analysis of single-strand breaks in plasmid DNA did not show any agreement with their theoretical data. This indicates that the mechanism of cell death as a result of radiosensitization with gold nanoparticles is a complex biological process that cannot be measured by a single parameter. McMahon et al also suggest that there is reduced radiosensitization in the megavoltage range of energy compared with the kilovoltage range of energy, but the reduction is not as great as that predicted theoretically. ${ }^{19}$ Variation in biological processes may contribute to the level of radiosensitization in the megavoltage range of energy, as indicated in the literature. ${ }^{4,17}$ The data indicate significant changes in the alpha value, while minimal variations are observed in the beta parameter. Similar results were obtained by Lechtman et al using Monte Carlo simulations. ${ }^{22}$ Further measurements focusing on this variation between two radiobiological parameters are currently being undertaken by our group.

Lechtman et al found that megavoltage energy could not achieve significant radiosensitization unless a higher 


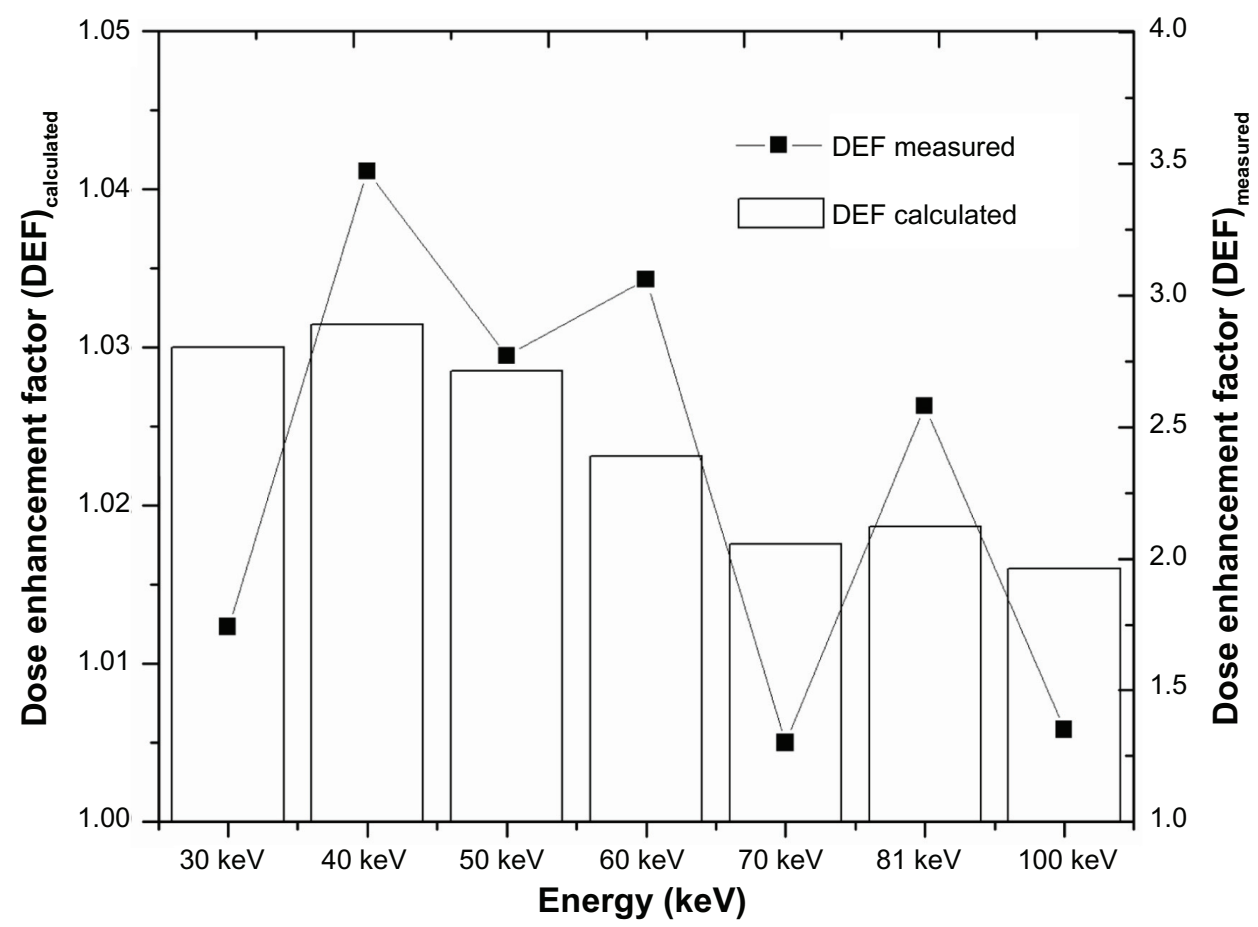

Figure 4 Comparison of calculated and experimental DEF for a mass concentration of gold nanoparticles of $0.197 \mathrm{mg} / \mathrm{mL}$ or a molar concentration of I mmol/L. Abbreviation: DEF, dose enhancement factor.

concentration of gold nanoparticles was used. ${ }^{15}$ Calculation of the proportion of energy transferred to escaping secondary charged particles or internally absorbed in the nanoparticles suggests that use of photon energy below the K-edge takes advantage of an extremely localized Auger cascade. Alternatively, implementation of high energy (above the K-edge) photons requires a high concentration of gold nanoparticles in the tumor region to acquire enough radiosensitization from the

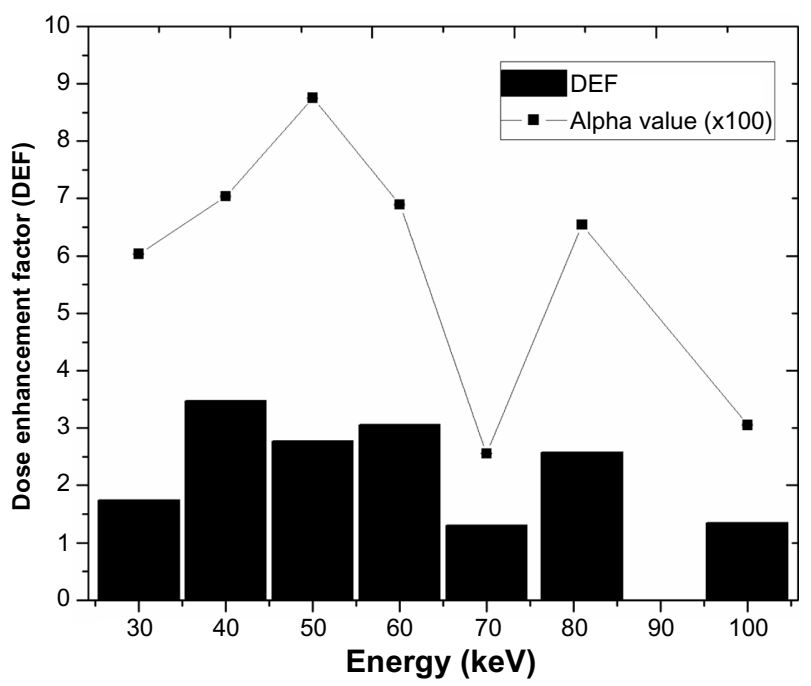

Figure 5 Correlation of the variation of X-ray energies with experimental DEF and alpha values.

Abbreviation: DEF, dose enhancement factor. energy deposited by photoelectrons. ${ }^{15}$ The localization of gold nanoparticles may not be an issue where photoelectric absorption becomes dominant. However, an increase in biologically lethal effects may be achieved if the gold nanoparticles can be internally targeted, especially to DNA. ${ }^{10}$ However, as previously noted, cell death could be induced by damage to other cell structures and suborganelles via a different molecular mechanism, especially in the presence of gold nanoparticles. ${ }^{21}$ Many studies have been reported that show many folds of radiosensitization being observed for kilovoltage energies which support this theory. ${ }^{1-3,5}$

The current literature reports conflicting results, and each study makes its own assumptions regarding the process of radiosensitization by gold nanoparticles in biological materials. Depending on the measurement end point, theoretical model, and analytical parameters used, the results can differ significantly. The clonogenic assay has been the gold standard for cell survival measurements and has been used in many radiobiological studies involving gold nanoparticles. ${ }^{4,5}$ Other methods have also been used to examine in detail the in vitro effects of gold nanoparticles in cells. For example, McMahon et al investigated the optimal energy for gold nanoparticles by using plasmid DNA and gamma H2AX foci to observe the DNA damage. ${ }^{4,19,23}$ It is very important to understand every part of the cellular effects and interaction with gold nanoparticles; however, the quantifying dose 
enhancement or radiosensitizing effect essentially arises from the total damage effects of the biological system. Therefore, more multiparametric approaches to measurement that take into account all the biological damage contributing to the radiosensitization effects must be carried out.

In optimizing the methods used for measuring radiosensitization in biological materials, polymer gel dosimetry could be a useful method to physically validate the dose enhancement effects produced by gold nanoparticles. A recent study of polymer gel dosimetry (normoxic polyacrylamide gel and radiochromic gel), show more dose enhancement in the kilovoltage range of energy than in the megavoltage range of energy. ${ }^{24,25}$ Comparison of the results for both types of gel is in close agreement, where the variation of DEF obtained for $6 \mathrm{mV}$ is 1.14 (polymer gel) and 1.11 (presage gel) while the DEF obtained at around $80 \mathrm{kV}$ energy is 1.76 (polymer gel) and 1.77 (presage gel). ${ }^{24,25}$ In contrast with the unpredictable behavior and variation of biological materials, polymer gel dosimetry provides a more controlled environment for the measurement of the physical dose enhancement effects of gold nanoparticles.

\section{Conclusion}

This study demonstrates the effects of radiation energy on the radiosensitization effects of gold nanoparticles on cells in culture. The results obtained clearly demonstrate that the cellular radiosensitization effects in the presence of gold nanoparticles are energy-dependent, and the experimental results are in close agreement with the theoretical calculations. The optimal energy was found to be around $40 \mathrm{keV}$, as predicted from the calculated ratio of the mass energy absorption coefficients for water and gold. The increase in deposited energy induced by gold nanoparticles may be closely related to cellular radiosensitization, but the relationship between them requires further investigation and consideration of multiple factors.

\section{Acknowledgments}

The authors acknowledge the technical support and beamtime allocations by the Japanese Synchrotron Radiation Research Institute. This work was supported by a grant from the Australian Synchrotron, CASS Foundation, RMIT University, and the Universiti Sains Malaysia Short Term Grant (304/PPSK/61312062).

\section{Disclosure}

All authors report no conflicts of interest in this work and that this research has no commercial value.

\section{References}

1. Hainfeld JF, Slatkin DN, Smilowitz HM. The use of gold nanoparticles to enhance radiotherapy in mice. Phys Med Biol. 2004;49:309-315.

2. Hainfeld JF, Dilmanian FA, Zhong Z, Slatkin DN, Kalef-Ezra JA, Smilowitz HM. Gold nanoparticles enhance the radiation therapy of a murine squamous cell carcinoma. Phys Med Biol. 2010;55: 3045-3059.

3. Rahman WN, Bishara N, Ackerly T, et al. Enhancement of radiation effects by gold nanoparticles for superficial radiation therapy. Nanomedicine. 2009;5:136-142.

4. Chithrani DB, Jelveh S, Jalali F, et al. Gold nanoparticles as radiation sensitizers in cancer therapy. Radiat Res. 2010;173:719-728.

5. Butterworth KT, Coulter JA, Jain S, et al. Evaluation of cytotoxicity and radiation enhancement using $1.9 \mathrm{~nm}$ gold particles: potential application for cancer therapy. Nanotechnology. 2010;21:295101.

6. Adams FA, Norman A, Mello RS, Bass D. Effects of radiation and contrast media on chromosomes Radiology. 1977;124:823-826.

7. Norman A, Adam FH, Riley RF. Cytogenetic effects of contrast media and triodobenzoic acids derivatives in human lymphocytes. Radiology. 1978;129:199-203.

8. Matsudaira H, Ueno AM, Furuno I. Iodine contrast medium sensitizes cultured mammalian cells to $\mathrm{X}$ rays but not to $\gamma$ rays. Radiat Res. 1980;84:144-148.

9. Mello RS, Callisen H, Winter J, Kagan AR, Norman A. Radiation dose enhancement in tumours with iodine. Med Phys. 1983;10:75-78.

10. Corde S, Joubert A, Adam JF, et al. Synchrotron radiation based experimental determination of the optimal energy for cell radiotoxicity enhancement following photoelectric effect on stable iodinated compounds. Br J Cancer. 2004;91:544-551.

11. Gastaldo J, Boudou C, Lamalle L, et al. Normoxic polyacrylamide gel doped with iodine: response versus X-ray energy. Eur J Radiol. 2008;68(Suppl 3):S118-S120.

12. Renier M. The radiotherapy clinical trials projects at the ESRF: technical aspects. Eur J Radiol. 2008;68(Suppl 3):S147-S150.

13. Cho SH. Estimation of tumour dose enhancement due to gold nanoparticles during typical radiation treatments: a preliminary Monte Carlo study. Phys Med Biol. 2005;50:163-173.

14. Cho SH, Jones BL, Krisnan S. The dosimetric feasibility of gold nanoparticle-aided radiation therapy (GNRT) via brachytherapy using low-energy gamma-/X-ray sources. Phys Med Biol. 2009;54: 4889-4905.

15. Lechtman E, Chattopadhyay N, Cai Z, Mashouf S, Reilly R, Pignol JP. Implications on clinical scenario of gold nanoparticle radiosensitization in regards to photon energy, nanoparticle size, concentration and location. Phys Med Biol. 2011;56:4631-4647.

16. Butterworth KT, McMahon SJ, Currell FJ, Prise KM. Physical basis and biological mechanisms of gold nanoparticles radiosensitization. Nanoscale. 2012;4:4830-4838.

17. Jain S, Coulter JA, Hounsell AR, et al. Cell specific radiosensitization by gold nanoparticles at megavoltage radiation energies. Int $J$ Radiat Oncol Biol Phys. 2011;79:531-539.

18. Berger MJ, Hubbell JH, Seltzer SM, et al. 1998 XCOM: Photon Cross Sections NIST Standard Reference Database 8 (XGAM). Available from: http://physics.nist.gov/PhysRefData/Xcom/Text/XCOM.html. Accessed March 18, 2014.

19. McMahon SJ, Hyland WB, Brun E, et al. Energy dependence of gold nanoparticle radiosensitization in plasmid DNA. J Phys Chem. 2011;115:20160-20167.

20. Zygmanski P, Hoegele W, Tsiamas P, et al. A stochastic model of cell survival for high-Z nanoparticle radiotherapy. Med Phys. 2013;40:024102.

21. Somosy Z. Radiation response of cell organelles. Micron. 2000;31: 165-181.

22. Lecthman E, Mashouf S, Chattopadhyay N, et al. A Monte Carlobased model of gold nanoparticle radiosensitization accounting for increased radiobiological effectiveness. Phys Med Biol. 2013;58: 3075-3087. 
23. Ngwa W, Korideck H, Kassis AI, et al. In vitro radiosensitization by gold nanoparticles during continous low dose rate gamma irradiation with I-125 brachytherapy seeds. Nanomedicine. 2013;9:25-27.

24. Alqathami M, Blencowe A, Un JY, Doran SJ, Qiao G, Geso M. Novel multicompartmental 3-dimensional radiochromic radiation dosimeters for nanoparticle-enhanced radiation therapy dosimetry. Int $J$ Radiat Oncol Biol Phys. 2012;84:549-555.
25. Rahman WN, Wong CJ, Ackerly T, Yagi N, Geso M. Polymer gel impregnated with gold nanoparticles implemented for measurements of radiation dose enhancement in synchrotron and conventional radiotherapy type beams. Australas Phys Eng Sci Med. 2012;35: 301-309.

\section{Publish your work in this journal}

The International Journal of Nanomedicine is an international, peerreviewed journal focusing on the application of nanotechnology in diagnostics, therapeutics, and drug delivery systems throughout the biomedical field. This journal is indexed on PubMed Central, MedLine, CAS, SciSearch $\AA$, Current Contents ${ } /$ Clinical Medicine,
Journal Citation Reports/Science Edition, EMBase, Scopus and the Elsevier Bibliographic databases. The manuscript management system is completely online and includes a very quick and fair peer-review system, which is all easy to use. Visit http://www.dovepress.com/ testimonials.php to read real quotes from published authors.

Submit your manuscript here: http://www.dovepress.com/international-journal-of-nanomedicine-journal 